\title{
Evidence for the return meridional flow in the convection zone from latitude motions of sunspots
}

\author{
K.R. Sivaraman ${ }^{1}$, H.Sivaraman ${ }^{2}$, S.S.Gupta ${ }^{3}$ and R.F.Howard ${ }^{4}$ \\ ${ }^{1}$ 5020, Haven Place, \#206, Dublin, CA-94568, USA and formerly of the Indian Institute of \\ Astrophysics, Bangalore, India. \\ email: kr_sivaraman@yahoo.com \\ ${ }^{2}$ 2581, Rivers Bend Circle, Livermore, CA-94550, USA. \\ ${ }^{3}$ Formerly of the Indian Institute of Astrophysics, Kodaikanal, India. \\ ${ }^{4}$ National Solar Observatory, Tucson, Arizona, USA.
}

\begin{abstract}
We have derived the latitude motions of sunspots classified into three area categories using the measures of positions and areas of their umbrae from the white - light images of the Sun for the period 1906 - 1987 from the Kodaikanal Observatory archives. The latitude motions are directed equator - ward in all the three area classes. We interpret that these equator ward latitude motions reflect the meridional flows at the three depths in the convection zone where the magnetic flux loops of the spots of the three area classes are anchored. We obtain estimates of the anchor depths through a comparison of the rotation rates of the spots in each area class with the rotation rate profiles from helioseismic inversions. The equator - ward flows measured by us thus provide evidence of the return meridional flows in the convection zone as required in the flux transport solar dynamo models. We have done an identical analysis using a similar data set derived from the photoheliogram collections of the Mt.Wilson Observatory for the period $1917-1985$. There is good agreement between the results from the data sets of the two observatories.
\end{abstract}

Keywords. Latitude motions, meridional flow, convection zone, flux transport

\section{Introduction}

The flux - transport model of the solar dynamo (the most successful among solar dynamo models) invokes meridional circulation (one cell each in the north and south hemispheres) consisting of a pole - ward flow on the surface and an equator - ward return flow in the interior, in addition to differential rotation (the $\Omega$ - effect) and helical turbulence (the $\alpha$-effect) in the dynamo mechanism in the $(\alpha-\Omega)$ type dynamos (Wang, Sheeley \& Nash 1991; Choudhuri, Schüssler \& Dikpati 1995; Charbonneau 2005). While the meridional flow on the surface is well known since a long time, the return flow, a key ingredient in the model has not been detected observationally so far (Charbonneau 2005; Dikpati 2005). Studies have shown that the rotation rates measured using sunspots as tracers reflect the rotation rates of the plasma layers in the interior at the respective depths where the foot points of the magnetic flux loops of spots of different ages (or areas) are anchored and not the surface rotation rate (Nesme-Ribes, Ferreira \& Mein 1993; Collin et al. 1995; Beck 2000; Sivaraman et al. 2003). Based on this scenario we have estimated the velocity of meridional flows at three depths in the convection zone by measuring the latitudinal drifts of spots divided into three area classes, assuming that spots of each area class reflect the latitudinal motion at the respective depths where their foot points are anchored, just as their rotation rates do. We matched the rotation rates 
of the spots in each area class (the same sample of spots divided into the same area classes as was used for deriving the meridional velocities) with helioseismic profiles ( plot of rotation rate $v s$ depth as $\frac{r}{R_{0}}$; r, radial distance and $R_{o}$ solar radius) and obtained estimates of the respective anchor depths in the convection zone.

\section{Data and analysis}

\subsection{Measurements:}

Our data consists of measures of position (heliographic latitude and longitude) and umbral area of every spot that has appeared on the disc within longitudes $\pm 60^{\circ}$ from the daily photoheliogram for the period 1906 - 1987 of the Kodaikanal Observatory, with a digitizing pad of spatial resolution of $0.02 \mathrm{~mm}$ that translates to $\approx 0.02$ arc sec on the $20 \mathrm{~cm}$. diameter solar image. Besides, we accessed a similar data set (created from the Mt.Wilson daily photoheliograms covering the period 1917 - 1985) from the NOAA site (ftp: //ftp.ngdc.noaa.gov/ STP/SOLAR-DATA/SUNSPOT-REGION-TILT). The present analysis is on the same lines as the one adopted for determining meridional flow velocities and anchor depths of spot groups (Sivaraman et al. 2010).

\subsection{Latitudinal drifts, meridional velocities and anchor depths:}

We computed the latitudinal drift (in deg day ${ }^{-1}$ ) of each spot by dividing the drift by the time elapsed (expressed in days) between successive observations. After eliminating spots with latitudinal drifts $\geqslant 1.5 \mathrm{deg}_{\text {day }}{ }^{-1}$ (equivalent to $\approx 200 \mathrm{~m} \mathrm{~s}^{-1}$ ) so as to minimize the errors, we had 118760 spots in the Kodaikanal data and 107020 spots in the Mt.Wilson data. To study the variation of latitudinal drifts with latitude we divided the latitude zone $+40^{\circ}$ to $-40^{\circ}$ into $5^{\circ}$ latitude zones and assigned the latitudinal drifts to that $5^{\circ}$ zone based on the initial latitudes of the spots. In the next step, we sorted the spots in the $5^{\circ}$ zones into three area classes - of umbral areas $0-5 \mu, 5-10 \mu$ and $>10 \mu$ ( $\mu$ area in millionths of the hemisphere) and computed the mean latitudinal drifts of spots of each area class in all of the $5^{\circ}$ zones. We multiplied the latitudinal drifts in deg day ${ }^{-1}$ by the factor of $140.596 \cos (\lambda)$ to convert to meridional velocities in $\mathrm{m} \mathrm{s}^{-1}$. Accordingly, a drift of $0.01 \mathrm{deg}$ day $^{-1}$ would correspond to a velocity of $\approx 1.3 \mathrm{~m} \mathrm{~s}^{-1}$ at $15^{\circ}$ latitude.

To estimate the anchor depths, we divided 118760 spots (Kodaikanal data) into three area classes $(0-5 \mu, 5-10 \mu$ and $>10 \mu)$ and computed the sidereal rotation rates of spots in each class and the least square solution for the latitude dependence as in Gupta, Sivaraman \& Howard 1999.

We projected the mean rotation rates of spots of each area class one after the other on the rotation rate vs depth $\left(\frac{r}{R_{0}}\right)$ profiles at five latitudes $\left(0^{\circ}, 10^{\circ}, 20^{\circ}, 30^{\circ}\right.$ and $\left.40^{\circ}\right)$ and read off the depths on the $\frac{r}{R_{0}}$ axis corresponding to the intercepts. These represent the anchor depths at the five latitudes and their mean, the mean anchor depth of spots of each area class. The internal rotation profiles are from the Global Oscillation Network Group (GONG ) data (Antia, 2008, private communication). We repeated the above for the 107020 spots in the Mt. Wilson data.

\section{Results and conclusions}

We show the variation of latitude drifts with latitude in Figure 1 and the values of latitudinal drifts ( $\operatorname{deg} \mathrm{day}^{-1}$ ) and the corresponding meridional velocities (in $\mathrm{m} \mathrm{s}^{-1}$ ) in Table 1 extracted from Figure 1, for the Kodaikanal and Mt.Wilson data.

- Within the latitude zone $+30^{\circ}$ to $-30^{\circ}$, the meridional flow is equator - ward in the three area classes (see Figure 1 and Table 1). This flow provides evidence for the 

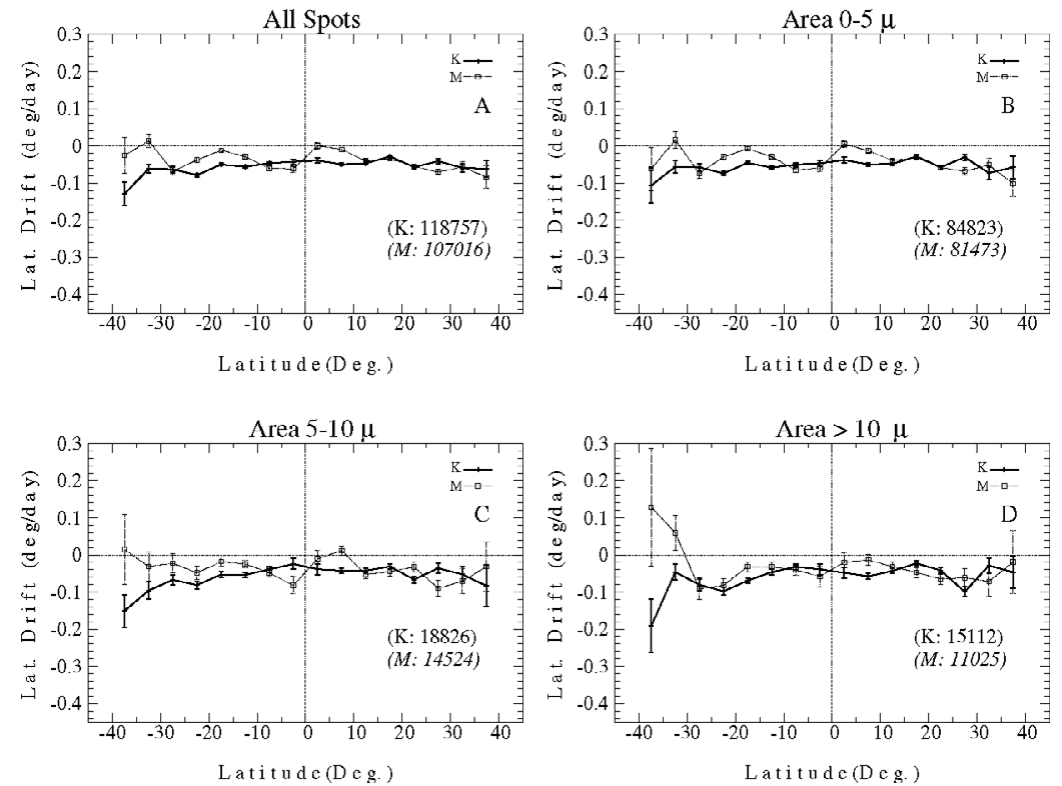

Figure 1. Latitudinal drifts (in deg day ${ }^{-1}$ ) averaged over the spots within five degree latitude bins vs. latitude from Kodaikanal white - light images for the period $1906-1987$ (K - and Mt. Wilson white - light images for the period $1917-1985(\mathbf{M} \cdots \bullet \cdots)$. The latitude of the spot at the time of initial observation defines the five-degree latitude zone (bin) to which it is assigned. Negative drifts indicate equator - ward motion in both hemispheres. The number of spots used for determining the latitudinal drifts in the respective area category is shown at the bottom right within brackets in each panel (prefix K stands for Kodaikanal data); similar number in italics with prefix $M$ stands for the number of spots of Mt. Wilson data.

Table 1. Latitudinal drift $\left(\mathrm{deg} \mathrm{day}^{-1}\right)$ and the equivalent meridional flow velocity (in $\mathrm{m} \mathrm{s}^{-1}$ ) averaged over $0^{\circ}-30^{\circ}$ latitude for the northern and southern hemispheres for the three area classes. (in bold: Kodaikanal data; in italics: Mt. Wilson data). Negative latitudinal drifts represent equator - ward motions. The mean anchor depth of spots of each area class is shown in the last column (see text).

\begin{tabular}{|c|c|c|c|c|c|}
\hline \multirow{2}{*}{$\begin{array}{l}\text { Area class } \\
\text { in } \mu\end{array}$} & \multicolumn{2}{|c|}{ North } & \multicolumn{2}{|c|}{ South } & \multirow{2}{*}{$\begin{array}{c}\text { Mean anchor } \\
\text { depth }\left(\frac{r}{R_{0}}\right)\end{array}$} \\
\hline & deg day ${ }^{-1}$ & $\mathrm{~m} \mathrm{~s}^{-1}$ & deg day ${ }^{-1}$ & $\mathrm{~m} \mathrm{~s}^{-1}$ & \\
\hline \multirow[t]{2}{*}{$<5$} & $-0.042 \pm 0.002$ & $5.7 \pm 0.3$ & -0 . & $7.5 \pm 0.4$ & 0.89 \\
\hline & $-0.034 \pm 0.003$ & $4.6 \pm 0.4$ & $-0.044 \pm 0.003$ & $6.0 \pm 0.4$ & 0.89 \\
\hline \multirow[t]{2}{*}{$5-10$} & $-0.043 \pm 0.004$ & $5.8 \pm 0.5$ & $-0.053 \pm 0.004$ & $7.2 \pm 0.5$ & 0.84 \\
\hline & $-0.036 \pm 0.006$ & $4.9 \pm 0.8$ & $-0.041 \pm 0.007$ & $5.6 \pm 0.9$ & 0.85 \\
\hline \multirow[t]{2}{*}{$>10$} & $-0.052 \pm 0.004$ & $7.1 \pm 0.5$ & $-0.061 \pm 0.004$ & $8.3 \pm 0.5$ & 0.78 \\
\hline & $-0.040 \pm 0.007$ & $5.4 \pm 0.9$ & $-0.056 \pm 0.008$ & $7.6 \pm 1.1$ & 0.78 \\
\hline \multirow[t]{2}{*}{ All spots } & $-0.044 \pm 0.002$ & $6.0 \pm 0.3$ & $-0.056 \pm 0.002$ & $7.6 \pm 0.3$ & Depth cannot \\
\hline & $-0.035 \pm 0.002$ & $4.8 \pm 0.3$ & $-0.045 \pm 0.003$ & $6.1 \pm 0.4$ & be assigned \\
\hline
\end{tabular}

return meridional flow in the convection zone that has remained undetected so far. This together with the surface pole - ward flow would act as a conveyor belt to transport the magnetic flux for recycling by the dynamo as envisaged in the flux - transport models.

- The equator - ward drift reaches high values in both hemispheres $(\approx-0.08$ to -0.09 deg day ${ }^{-1}$ or 10 to $12 \mathrm{~m} \mathrm{~s}^{-1}$ ) around latitude $\pm 25^{\circ}$ and slows down towards zero drift near the equator and at latitudes $\pm 35^{\circ}$ (see Figure 1). One or two such high values result in increase in the mean velocities particularly for large spots (area $>10 \mu$ ). Chou 
\& Dai (2001) analyzing the images from the Taiwan Oscillation Network for the period 1994 - (2001) with time - distance helioseismology technique noticed that at depths $>10$ $\mathrm{Mm}$, a new component of meridional flow diverging from the activity latitudes $\left(\approx 25^{\circ}\right)$ appeared in both hemispheres as the solar activity developed during $1998-2000$. We are of the view that the surge in meridional velocity in the same latitude zone seen in our study is the signature of the divergent flow noticed by Chou \& Dai (2001). Our analysis, in addition shows that this divergent flow is stronger for spots of large areas (hence large magnetic fields). In an earlier study it was shown that spot groups show similar equatorward flow (Sivaraman et al. 2010). But the divergent flow was not large enough to cause an overall increase in the velocity as in the case of individual spots. The reason perhaps is that group motions (because the amplitudes are so small) are influenced by the possibly random appearance and disappearance of spots in the group, which shifts the calculated position of the group. Thus there is a cause unrelated to meridional motion that affects group meridional motion. This uncertainty being absent in the case of individual spots, we are able to detect the small and subtle changes in their latitudinal motions.

\section{Acknowledgements}

The presentation of this paper in the IAU Symposium 273 was possible due to partial support from the National Science Foundation grant numbers ATM 0548260, AST 0968672 and NASA - Living With a Star grant number 09-LWSTRT09-0039. One of the authors (KRS) wishes to thank the LOC, in particular Dr. Debi Prasad Choudhary and Dr. Cristina Cadavid. We wish to acknowledge the sincere efforts of several generations of observers at the Kodaikanal and Mt. Wilson Observatories who made these observations covering many decades. Without their very dedicated and careful work this study would not have been possible.

\section{References}

Beck, J. G.: 2000, Solar Phys.191, 47.

Charbonneau, P.: 2005, "Dynamo Models of the Solar Cycle": In Living Reviews in Solar Physics. Irsp $-2005-2$.

Chou, D.-Y. \& Dai, D.-C: 2001, Astrophys. J. Lett. 559, L175.

Choudhuri, A. R. \& Schüssler, M., Dikpati.M.: 1995, Astron. Astrophys 303, L29.

Collin, B., Nesme-Ribes, E., Lereoy, B., Meunier, N., \& Sokoloff, D.: 1995, C.R. Acad. Sci. Paris; t. 321, Serie II.b, 111.

Dikpati, M.: 2005, Adv. Space Res. 35, 322.

Gupta, S. S., Sivaraman, K. R., \& Howard, R. F.: 1999, Solar Phys. 188, 225.

Nesme-Ribes, E., Ferreira, E. N., \& Mein, P.: 1993, Astron. Astrophys 274, 563.

Sivaraman, K. R., Sivaraman, H., Gupta, S. S., \& Howard, R. F.: 2003, Solar Phys. 214, 65.

Sivaraman, K. R., Sivaraman, H., Gupta, S. S., \& Howard, R. F.: 2010, Solar Phys. 266, 247.

Wang, Y.-M., Sheeley, N. R., \& Nash, A. G.: 1991, Astrophys. J. 383, 431. 